\title{
The Relationship between VEGFA and TGFB1 Polymorphisms and Target Lesion Revascularization after Elective Percutaneous Coronary Intervention
}

\author{
Tadeusz Osadnik, ${ }^{1}$ Andrzej Lekston, ${ }^{2}$ Kamil Bujak, ${ }^{2}$ Joanna Katarzyna Strzelczyk, ${ }^{3}$ \\ Lech Poloński, ${ }^{2}$ and Mariusz Gąsior ${ }^{2}$ \\ ${ }^{1}$ 2nd Department of Cardiology and Angiology, Silesian Center for Heart Disease, Zabrze, Poland \\ ${ }^{2} 3 r d$ Department of Cardiology, School of Medicine with the Division of Dentistry in Zabrze, Medical University of Silesia in Katowice, \\ Silesian Center for Heart Disease, Zabrze, Poland \\ ${ }^{3}$ Department of Medical and Molecular Biology, School of Medicine with the Division of Dentistry in Zabrze, Medical University of \\ Silesia, Katowice, Poland
}

Correspondence should be addressed to Tadeusz Osadnik; tadeusz.osadnik@sccs.pl

Received 23 January 2017; Revised 10 June 2017; Accepted 14 June 2017; Published 24 July 2017

Academic Editor: Michele Malaguarnera

Copyright (c) 2017 Tadeusz Osadnik et al. This is an open access article distributed under the Creative Commons Attribution License, which permits unrestricted use, distribution, and reproduction in any medium, provided the original work is properly cited.

Background and Aim. The specific association between genetic variation and in-stent restenosis is still only partly understood. The aim of this study is to analyze the relationship between functional polymorphisms in the genes encoding vascular endothelial growth factor A (VEGF-A; rs699947) and transforming growth factor beta 1 (TGF- $\beta 1$; rs1800470) and target lesion revascularization (TLR) risk. Methods. A total of 676 patients (805 lesions) with stable coronary artery disease (SCAD) who received elective percutaneous coronary intervention (PCI) with at least one bare-metal stent implantation were included. The primary study endpoint was TLR at a 4-year follow-up. Results. The TLR rate was higher in patients with the VEGFA A/A genotype $(15.4 \%)$ than in patients with the VEGFA A/C (7.9\%) and $\mathrm{C} / \mathrm{C}(8.9 \%)$ genotypes $(p=0.009)$. The VEGFA A/A genotype, after adjustment for clinical and procedural covariates, remained significantly and independently associated with the TLR (hazard ratio-2.09 [95\% confidence interval 1.32-3.33, $p=0.0017]$ ). However, we found no association between TLR and the TGFB1 genotype. Conclusion. The VEGFA A/A genotype is significantly and independently associated with TLR risk in Polish SCAD patients who received elective PCI with bare-metal stent implantation.

\section{Background}

In-stent restenosis (ISR) is a major limitation of percutaneous coronary intervention (PCI). To date, the etiology and genetic basis of this phenomenon are only partly understood. A wide array of inflammatory cytokines, growth factors, and mitogens as well as abnormal regional wall shear stress leads to intimal hyperplasia [1-6]. The authors hypothesize that polymorphisms in specific genes generate individual differences in the vascular wound healing process in response to wall injury after stent implantation.

Experimental studies have proven the important role of vascular endothelial growth factor A (VEGF-A) and transforming growth factor beta 1 (TGF- $\beta 1$ ) in the formation of neointima and ISR development [7]. Nonetheless, the relationship between polymorphisms in genes encoding these growth factors and target lesion revascularization (TLR) risk has not been analyzed before. Therefore, we aimed to determine whether the polymorphisms in VEGFA (rs699947) and TGFB1 (rs1800470) are associated with TLR in a prospective, population-based cohort of Polish patients who underwent PCI with bare-metal stent (BMS) implantation. We chose rs699947 and rs1800470 as previous studies confirmed that they are functional polymorphisms and affect both gene expression and VEGF-A and TGF- $\beta 1$ serum levels $[8,9]$. Moreover, we previously 
reported that rs699947 and rs1800470 polymorphisms in the genes encoding VEGF-A and TGF- $\beta 1$, respectively, are associated with late lumen loss (LLL) in patients with stable coronary artery disease (CAD) who received elective PCI with BMS implantation [10]. Additionally, these polymorphisms have been studied in the context of other cardiovascular disorders and have been proven to affect, inter alia, the angiographic severity of CAD [11, 12] and the risk of myocardial infarction $[13,14]$.

\section{Methods}

2.1. Patient Population. We enrolled 676 Caucasian patients (805 lesions) with stable CAD who underwent elective PCI between January 2007 and December 2012 with the implantation of at least one BMS. The patients were not related to each other. The primary study endpoint was TLR at a 4year follow-up, which was defined as either repeat percutaneous revascularization for a lesion anywhere within the stent, within $5 \mathrm{~mm}$ of the previously implanted stent, or the need for surgical revascularization of the stented vessel due to ISR. During index hospitalization, demographic and clinical data as well as periprocedural variables were recorded, including stent localization and diameter, the total stent length per lesion, and the number of stents implanted. Follow-up data on TLR were collected during subsequent hospitalizations. The survival information was based on a National Health Fund insurance status because a National Health Fund insurance policy is obligatory for all Polish citizens $[15,16]$.

2.2. Genotyping. DNA was extracted from blood samples using the GeneMATRIX Quick Blood DNA Purification Kit (EURX, Poland) according to the manufacturer's instructions. Similar to our previously used methods [10, 17], we identified single-nucleotide polymorphisms (SNPs) in the TGFB1 and VEGFA genes using TaqMan genotyping assays on the 7300 Real-Time PCR System and the SDS 1.4 Allelic Discrimination software (Applied Biosystems, USA). Samples that were initially identified as homozygous and heterozygous were sequenced, and after genotype confirmation, they were used as positive controls. DNase-, RNase-, and protease-free water (Qiagen, Germany) was used as the negative control. For quality control, $10 \%$ of the samples were randomly repeated and showed complete agreement.

2.3. Statistical Analysis. Continuous variables are reported as the mean \pm standard deviation. Categorical variables are presented as percentages. The chi-square test was used to determine whether the analyzed genotypes agreed with the Hardy-Weinberg equilibrium. Minor allele frequencies were calculated and reported. The Kaplan-Meier method was used to study the cumulative incidence of TLR overtime, whereas the log-rank test was applied to evaluate differences between patients with different VEGFA and TGFB1 genotypes using the dominant model (homozygous major versus heterozygous and homozygous minor) and codominant model setting (homozygous major versus heterozygous versus homozygous
TABLE 1: Baseline clinical and procedural characteristics.

\begin{tabular}{|c|c|c|}
\hline \multicolumn{3}{|c|}{ Clinical characteristics ( $n=676$ patients) } \\
\hline \multicolumn{2}{|l|}{ Age (years) } & $63.4 \pm 9.3$ \\
\hline \multicolumn{2}{|l|}{ Female } & $194(28.7)$ \\
\hline \multicolumn{2}{|l|}{ Hypertension } & $467(69.1)$ \\
\hline \multicolumn{2}{|c|}{ Diabetes mellitus } & $181(26.8)$ \\
\hline \multicolumn{2}{|c|}{ Previous myocardial infarction } & $369(54.6)$ \\
\hline \multicolumn{2}{|c|}{ Atrial fibrillation } & $89(13.1)$ \\
\hline \multicolumn{2}{|l|}{ Previous PCI } & $277(41)$ \\
\hline \multicolumn{2}{|l|}{ Previous CABG } & $66(9.8)$ \\
\hline \multicolumn{2}{|c|}{ Creatinine $(\mu \mathrm{mol} / \mathrm{l})$} & $84.3 \pm 34.1$ \\
\hline \multicolumn{3}{|c|}{ Procedural characteristics ( $n=805$ lesions) } \\
\hline \multicolumn{3}{|c|}{ LM } \\
\hline \multirow{4}{*}{ Vessel treated } & LAD & $220(27.3)$ \\
\hline & $\mathrm{Cx}$ & $257(31.9)$ \\
\hline & RCA & $300(37.3)$ \\
\hline & SVG & $13(1.6)$ \\
\hline \multicolumn{2}{|l|}{ Ostial lesion } & $19(2.4)$ \\
\hline \multicolumn{2}{|c|}{ Bifurcation lesion } & $75(9.3)$ \\
\hline \multicolumn{2}{|c|}{$\begin{array}{l}\text { Number of stents implanted per } \\
\text { lesion }\end{array}$} & $1.1 \pm 0.34$ \\
\hline \multicolumn{2}{|c|}{ Total stent length per lesion (mm) } & $19.5 \pm 9.4$ \\
\hline \multicolumn{2}{|c|}{ Minimal stent diameter (mm) } & $3.01 \pm 0.54$ \\
\hline \multicolumn{2}{|l|}{ Predilatation } & $412(51.2)$ \\
\hline \multicolumn{2}{|l|}{ Postdilatation } & $72(8.9)$ \\
\hline
\end{tabular}

Continuous variables are presented as the mean \pm standard deviation. Categorical variables are presented as number of patients/lesions (percentages). PCI: percutaneous coronary intervention; CABG: coronary artery bypass grafting; LM: left main; LAD: left anterior descending; $\mathrm{Cx}$ : circumflex branch; RCA: right coronary artery; SVG: saphenous vein graft.

minor). Patients who died before TLR occurred were censored at the time of death. To adjust for baseline clinical and periprocedural variables after positive evaluation of the proportional hazards assumption, Cox regression analysis was performed using TLR as a dependent variable. Prior to Cox regression analysis, the missing values were replaced using a state-of-the-art statistical method for mixed-type data imputation, the MissForest algorithm, to minimize information loss and the necessity to exclude entire cases due to single missing values. The MissForest algorithm is considered superior to other multiple imputation methods such as multivariable imputation by chained equations (MICE) [18]. Every variable, including the outcome variable, was included in the multiple imputation algorithm. To visualize the impact of the VEGFA and TGFB1 genotypes on TLR, the adjusted Kaplan-Meier curves were plotted using the inverse probability weight method and compared with the log-rank test for adjusted curves. To account for multiple comparisons resulting from multiple tests of VEGFA and TGFB1 SNPs using the dominant and codominant models, a Bonferroni-corrected $p$ value $=0.05 / 4=0.0125$ was considered statistically significant. Statistical analyses were entirely performed using $\mathrm{R}$ software and freely available statistical packages [19-21]. 
TABLE 2: Distribution of TGFB1 and VEGFA polymorphism genotypes in the analyzed patient cohort $(n=676)$.

\begin{tabular}{|c|c|c|c|c|c|c|}
\hline Gene/polymorphism & Homozygous major & Heterozygous & Homozygous minor & HWE $p$ value & MAF & MAF EU population [51] \\
\hline \multirow{3}{*}{ TGFB1 (rs1800470) } & $\mathrm{A} / \mathrm{A}$ & $\mathrm{A} / \mathrm{G}$ & $\mathrm{G} / \mathrm{G}$ & & & \\
\hline & $224(33.8 \%)$ & $310(46.8 \%)$ & $129(19.5 \%)$ & 0.27 & 42.8 & 38 \\
\hline & $\mathrm{A} / \mathrm{A}$ & $\mathrm{A} / \mathrm{C}$ & $\mathrm{C} / \mathrm{C}$ & & & \\
\hline VEGFA (rs699947) & $186(27.6 \%)$ & $322(47.7 \%)$ & $167(24.7 \%)$ & 0.25 & 48.6 & 50 \\
\hline
\end{tabular}

Genotypes of TGFB1 and VEGFA were successfully established for 663 (98.1\%) and 675 (99.9\%) patients, respectively. MAF: minor allele frequency; EU: European Union; HWE: Hardy-Weinberg equilibrium.

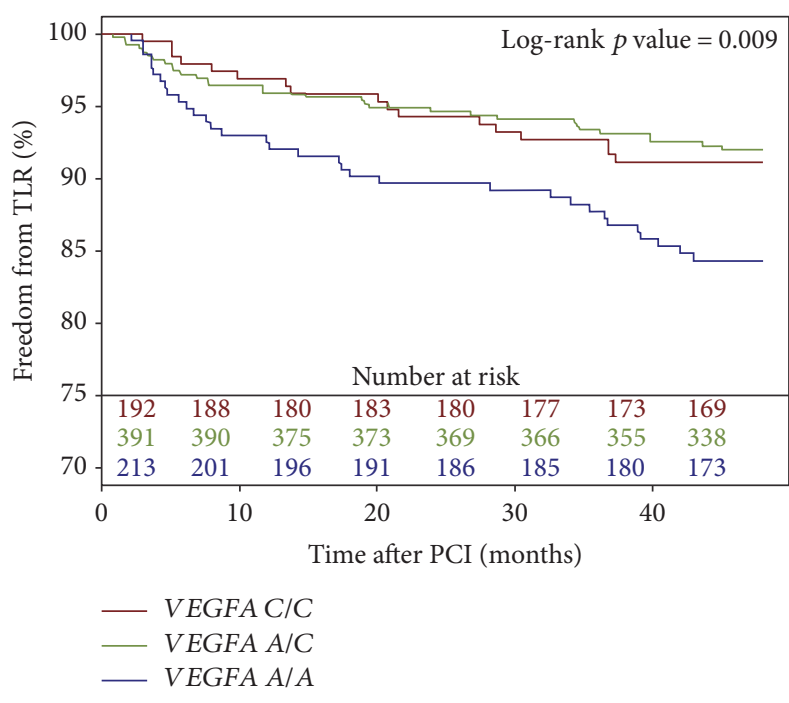

(a)

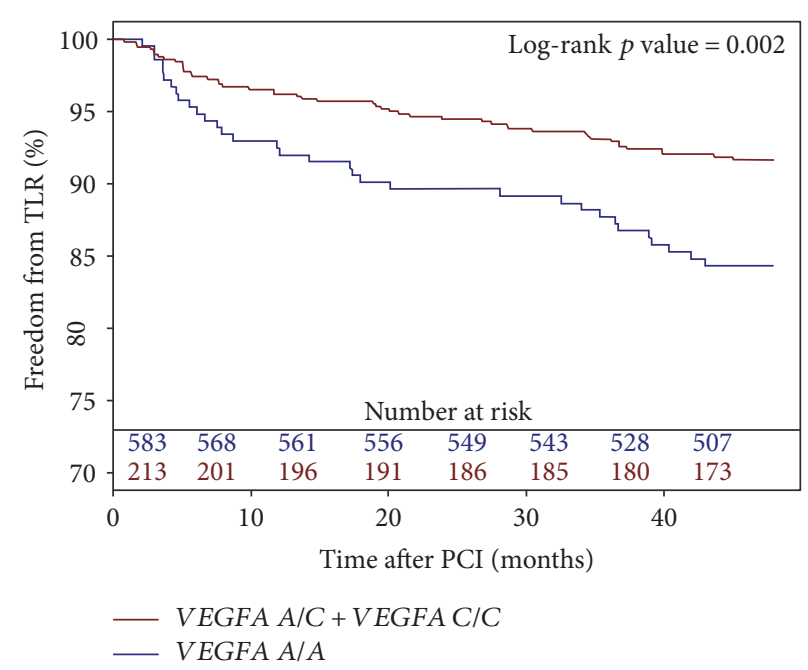

(b)

FIGURE 1: Freedom from TLR according to the VEGFA polymorphism genotypes using the codominant (a) and dominant model (b).

The study conformed to the Declaration of Helsinki and was approved by the Ethics Committee of the Silesian Medical Chamber in Katowice, Poland.

\section{Results}

Baseline clinical and periprocedural characteristics are presented in Table 1. The VEGFA and TGFB1 genotype distribution agreed with the Hardy-Weinberg equilibrium, and minor allele frequencies were similar to those reported for European populations (Table 2). Genotypes of TGFB1 and VEGFA were successfully established for 663 (98.1\%) and 675 (99.9\%) patients, respectively. The 48-month follow-up was available for 670 (99.1\%) patients. During the followup period, 25 (3.7\%) patients died. The TLR rate was higher in patients with the VEGFA A/A (15.4\%) genotype than in patients with the VEGFA A/C (7.9\%) and C/C (8.9\%) genotypes using the codominant (Figure 1(a)) and dominant models (Figure 1(b)) $(p=0.009$ and $p=0.002$, resp.). There were, however, no differences in TLR frequency for different TGFB1 genotypes (A/A-8.4\%, $\mathrm{A} / \mathrm{G}-11.2 \%$, and $\mathrm{G} /$ G-11.5\%) using the codominant (Figure 2(a)) and dominant (Figure 2(b)) models $(p=0.397$ and $p=0.175$, resp.). The VEGFA A/A genotype, after adjustment for clinical and periprocedural covariates, remained significantly and independently associated with TLR (adjusted log-rank $p=0.006$, hazard ratio (HR) -2.09 [95\% confidence interval (CI) $1.32-3.33, p=0.0017]$; Figures 3 (a) and 4 ), whereas no association was observed for the TGFB1 A/A genotype (ref. A/G and G/G; adjusted log-rank $p=0.186$, HR-0.67 [95\% CI $0.40-1.12, p=0.12$ ]; Figures $3(\mathrm{~b})$ and 4 ). Other factors associated with TLR were minimal stent diameter (HR (per $1 \mathrm{~mm}$ increase) -0.43 [95\% CI 0.26-0.7, $p=0.0008$ ]), stent length (HR (per $3 \mathrm{~mm}$ increase)-1.08 [95\% CI 1.03-1.13, $p=0.003]$ ), and lesions localized in the circumflex branch of the left coronary artery (reference LAD) (HR-0.49 [95\% CI 0.24-0.99, $p=0.04]$ ) (Figure 4).

\section{Discussion}

Atherosclerosis is considered a multifactorial disease influenced by environmental and genetic factors. Furthermore, the pathophysiological mechanisms of restenosis have also not yet been fully explained. Coronary angioplasty injures the arterial wall, leading to parietal thrombus formation and a local inflammatory response, which is considered the main driver of vascular smooth muscle cell (VSMC) proliferation and neointima formation [22]. VSMC proliferation is stimulated by the cytokines released from monocytes/macrophages [3], and studies have shown that one week after PCI, the neointima contains 60\% VSMCs and 30\% neutrophils and monocytes. In the subsequent weeks, the number of 


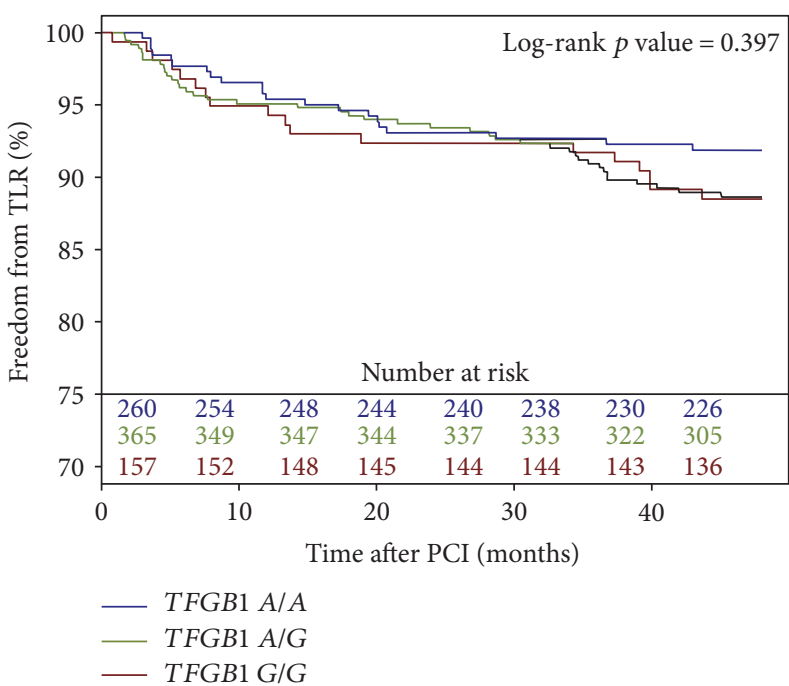

(a)

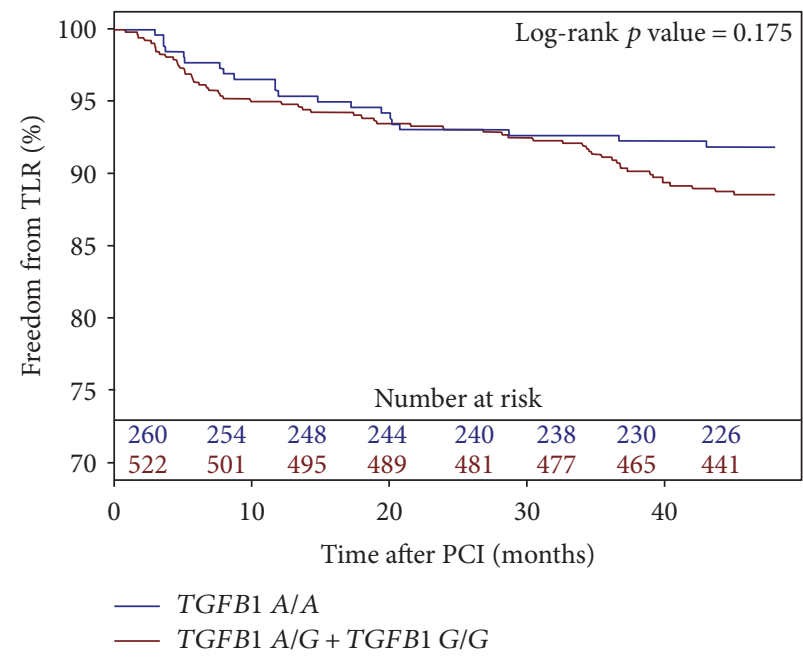

(b)

Figure 2: Freedom from TLR according to TGFB1 polymorphism genotypes using the codominant (a) and dominant model (b).

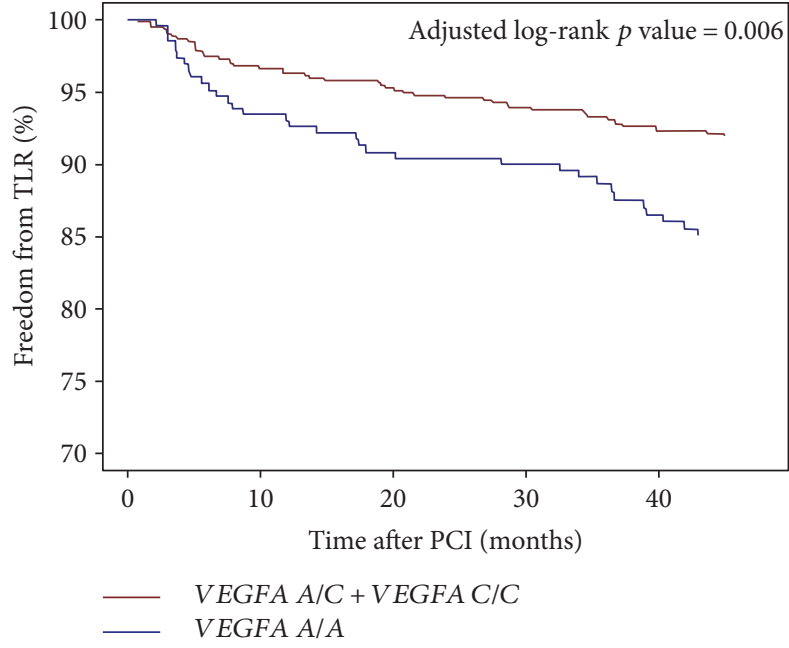

(a)

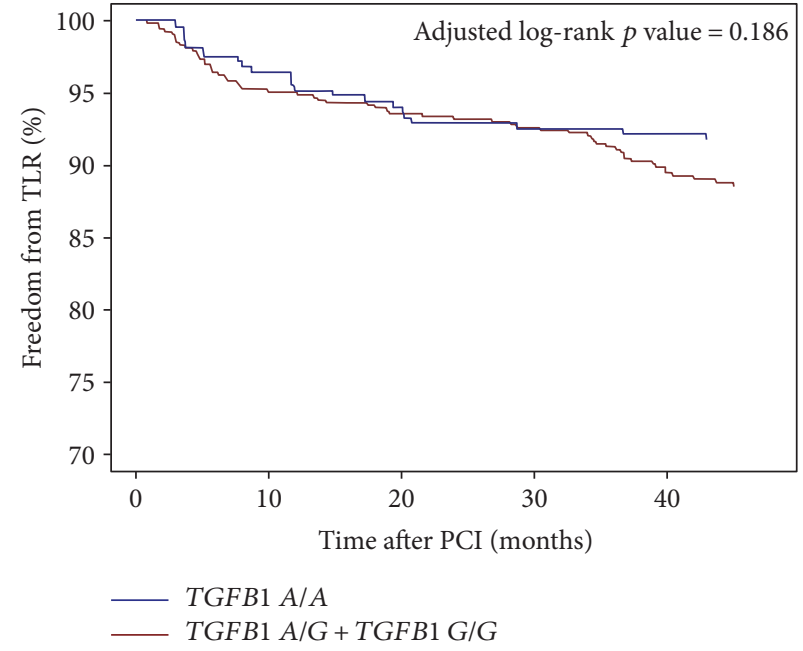

(b)

Figure 3: Freedom from TLR according to the VEGFA (a) and TGFB1 (b) polymorphism genotypes adjusted for clinical and periprocedural covariates.

mononuclear cells decreases, which is accompanied by a significant increase in the VMSC cell percentage. Four weeks after stent implantation, over $90 \%$ of the cells that form the neointima are VMSCs [23]. Therefore, many genetic studies on restenosis have examined genes encoding inflammation-related proteins, particularly polymorphisms in the genes for interleukin-1, interleukin-10, interleukin-1 receptor antagonist, matrix metalloproteinases, and C-reactive protein [24, 25]. Mediators of inflammation produced by monocytes and macrophages stimulate the release of interleukins, TGF- $\beta 1$, and other growth factors, specifically, VEGF-A. These proteins modulate the proliferative activity of endothelial cells and VSMCs and affect adhesion molecule expression [26, 27].
4.1. rs 1800470 Polymorphism (TGFB1). TGF- $\beta 1$ is a cytokine with a complex mechanism of action. TGF- $\beta 1$ mainly stimulates TGF- $\beta$ receptor type II (TGFBR2), which recruits TGF$\beta$ receptor type I (TGFBR1) to form a complex (TGF- $\beta 1$ + TGFBR2 + TGFBR1) that activates the Smad pathway. Throughout this pathway, TGF- $\beta 1$ exhibits antiproliferative [28] and anti-inflammatory effects [29], accelerates cell differentiation [30], and promotes extracellular matrix synthesis [31]. TGF- $\beta 1$ released locally by arterial wall fibroblasts sends paracrine signals to the VSMCs and the macrophages migrating toward the injured region of the stented arterial wall. The platelets that participate in parietal thrombus formation release large amounts of TGF- $\beta 1$ [32]; furthermore, serum TGF- $\beta 1$ levels might be genetically determined. 
Hazard ratio

Age (per 1 year)

Male

Hypertension

Previous MI

AF

Previous PCI

Creatinine (per $1 \mu \mathrm{mol} / \mathrm{l})$

Previous CABG

Ostial lesion

Bifurcation lesion

Stent length (per $3 \mathrm{~mm}$ )

Minimal stent diameter (per $1 \mathrm{~mm}$ )

Diabetes

PVD

MVD

Cx (ref. LAD)

LM (ref. LAD)

RCA (ref. LAD)

SVG (ref. LAD)

Predilatation

Postdilatation

VEGFA A/A (ref .VEGFA A/C \& C/C)

TGFB1 A/A (ref. TGFB1 A/G \& G/G)
0.980

0.91

0.69

0.93

1.38

1.30

1.002

1.84

1.38

0.95

1.07

0.83

1.06

1.09

1.06

0.49

2.63

1.65

1.84

1.27

0.71

2.12

0.67
Confidence interval

0.950-1.004

0.53-1.55

0.43-1.10

$0.56-1.57$

$0.76-2.53$

$0.76-2.22$

$1.000-1.008$

0.85-4.0

$0.47-4.11$

$0.41-2.20$

$1.02-1.12$

0.75-0.92

$0.63-1.78$

$0.44-2.70$

$0.57-1.96$

0.24-0.99

$0.60-11.6$

$0.95-2.90$

$0.47-7.25$

$0.80-2.03$

$0.30-1.67$

1.34-3.37

$0.40-1.12$ $p$-value

0.10

0.74

0.10

0.82

0.32

0.31

0.50

0.13

0.56

0.86

0.003

0.0008

0.80

0.87

0.86

0.05

0.17

0.08

0.39

0.31

0.42

0.002

0.12

FIGURE 4: Multivariate analysis of the impact of variables associated with clinical and periprocedural characteristics on 4-year TLR. TLR: target lesion revascularization; SCAD: stable coronary artery disease; PCI: percutaneous coronary intervention; BMS: bare-metal stent; MI: myocardial infarction; AF: atrial fibrillation; CABG: coronary artery bypass grafting; PVD: peripheral vascular disease; MVD: multivessel coronary disease; Cx: circumflex branch; LAD: left anterior descending; LM: left main; RCA: right coronary artery; SVG: saphenous vein graft. 
According to some studies, the presence of allele $\mathrm{C}$ correlates with higher TGF- $\beta 1$ concentrations [23-34]. Other researchers have observed increased TGF- $\beta 1$ levels in T/T genotype carriers [35]. TGF- $\beta 1$ inhibits proliferation in G1 phase, although at levels higher than 1-2 fg per cell, it might promote smooth muscle cell, fibroblast, and chondrocyte proliferation [36]. There is no linear correlation between TGF- $\beta 1$ expression and its effect on the cells in the restenosis process. Studies performed at our center have not shown a relationship between serum TGF- $\beta 1$ concentration and history of restenosis, including recurrent restenosis and the first restenosis [37, 38]. Recently, Chung et al. reported that blocking TGF- $\beta 1$ by intravascular local gene delivery does not reduce neointima formation but enhances the inflammatory response in a pig model of restenosis, which potentially aggravates lesion progression [39]. Previous studies indicated that the rs1800470 genotype is associated with the risk of developing cardiovascular diseases and their complications, inter alia, cerebral infarction [40], silent myocardial ischemia in diabetic patients [41], and CAD complications [13]. Yang et al. investigated the relationship between the rs 1800470 polymorphism and angiographic severity of CAD in Chinese population. They found that allele $\mathrm{T}$ is associated with higher CAD burden assessed using the Gensini Score [11]. On the other hand, a study conducted at our center did not confirm these findings in the population of Polish patients [17]. TGFB1 polymorphism (rs1800470) has been also studied in the context of ISR. Fragoso et al. reported, for the first time, that rs 1800470 polymorphism could be involved in the risk of developing ISR in the Mestizo population undergoing PCI with drug-eluting stent or BMS implantation [42]. In our previous study, we showed that the TGFB1 polymorphism (rs1800470) allele $\mathrm{T}$ is associated with decreased neointima formation in patients with CAD receiving BMS [10]. Any discrepancy with our previous study can be explained by the use of different inclusion criteria and the different endpoints of both studies.

4.2. rs699947 Polymorphism (VEGFA). VEGF-A is a potent and highly specific endothelial cell mitogen that regulates endothelial integrity [43-45], although in the literature, there is still a debate regarding whether VEGF-A is a proatherosclerotic or antiatherosclerotic factor [12]. rs699947 is a functional polymorphism associated with VEGF-A levels. In particular, Shahbazi et al. reported that the $\mathrm{C} / \mathrm{C}$ genotype is associated with higher VEGF-A synthesis than the A/A genotype [46]. Howell et al. genotyped 941 patients with CAD for the rs699947 polymorphism, and the A/A genotype frequency increased stepwise with the number of diseased coronary arteries using the $\mathrm{C} / \mathrm{C}$ genotype as the reference. Therefore, the A/A genotype is a risk factor for atherosclerosis, and the $\mathrm{C} / \mathrm{C}$ genotype is protective [12]. Our previous study evaluating the Gensini Score as a marker of atherosclerotic burden relative to SNPs revealed that the A/A genotype was more frequently observed than the $\mathrm{C} / \mathrm{C}$ genotype in patients with the highest Gensini Score [17]. Results of the meta-analysis of seven case-control studies indicated that rs699947 may be associated with the risk of CAD development, and A allele carriers have higher CAD susceptibility in comparison with the C allele carriers [47]. Furthermore, the rs699947 VEGFA polymorphism is associated with collateral circulation in CAD patients [48] and myocardial infarction risk in patients with rheumatoid arthritis [14] as well as may affect the antihypertensive responses to enalapril [49]. In our previous analysis, we showed that the A/A genotype is a risk factor for increased neointima formation, whereas the $\mathrm{C} / \mathrm{C}$ genotype was protective [10]. On the other hand, we did not find any relationship between VEGFA rs699947 and the risk of binary ISR [10]. Moreover, Bagyura et al. who analyzed the relationship between VEGFA polymorphisms and the risk of ISR in patients who underwent PCI with BMS implantation reported that rs699947 polymorphism is associated with neither the risk of diffuse nor focal ISR [50]. Our current analysis shows for the first time that the rs699947 A/A genotype is associated with a higher TLR risk. Similar to the results of the analysis of TGFB1 polymorphism, the discrepancies with previous studies regarding the role of VEGFA in ISR development could be associated with different inclusion criteria and different study endpoints.

4.3. Conclusions. In summary, we report for the first time that the rs699947 polymorphism in the VEGFA gene is associated with TLR in patients with stable CAD receiving PCI with stent implantation. This study suggests that a genetic polymorphism in VEGFA might be applicable to risk stratification for TLR. More detailed genetic studies in different ethnic populations are needed to further evaluate the association between VEGFA polymorphisms and ISR.

\section{Conflicts of Interest}

The authors declare that they have no competing interests.

\section{Acknowledgments}

This study was supported by the research Grants 2011/01/D/ NZ5/04387 and 2014/13/B/NZ5/03166 from the National Science Center, Poland.

\section{References}

[1] W. G. Feero, A. E. Guttmacher, C. J. O’Donnell, and E. G. Nabel, "Genomics of cardiovascular disease," The New England Journal of Medicine, vol. 365, no. 22, pp. 20982109, 2011.

[2] J. Wasilewski, T. Osadnik, S. Peryt Stawiarska, and L. Poloński, "The role of haemodynamic factors in the development of in-stent restenosis," Kardiologia Polska, vol. 70, no. 11, pp. 1194-1198, 2012.

[3] W. S. Weintraub, "The pathophysiology and burden of restenosis," The American Journal of Cardiology, vol. 100, no. 5, pp. 3K-9K, 2007.

[4] T. Osadnik, K. Bujak, and A. Lekston, "Is GLUT1 a potential target for in-stent restenosis treatment?" International Journal of Cardiology, vol. 223, pp. 199-200, 2016. 
[5] T. Osadnik, J. Strzelczyk, K. Bujak et al., "Functional polymorphism rs710218 in the gene coding GLUT1 protein is associated with in-stent restenosis," Biomarkers in Medicine, vol. 9, no. 8, pp. 743-750, 2015.

[6] T. Osadnik, J. K. Strzelczyk, M. Fronczek et al., "Relationship of the rs1799752 polymorphism of the angiotensin-converting enzyme gene and the rs699 polymorphism of the angiotensinogen gene to the process of in-stent restenosis in a population of Polish patients with stable coronary artery disease," Advances in Medical Sciences, vol. 61, no. 2, pp. 276-281, 2016.

[7] J. W. Jukema, J. J. W. Verschuren, T. A. N. Ahmed, and P. H. A. Quax, "Restenosis after PCI. Part 1: pathophysiology and risk factors," Nature Reviews Cardiology, vol. 9, no. 1, pp. 53-62, 2012.

[8] C.-C. Szeto, K.-M. Chow, P. Poon, C. Y.-K. Szeto, T. Y.-H. Wong, and P. K.-T. Li, "Genetic polymorphism of VEGF: impact on longitudinal change of peritoneal transport and survival of peritoneal dialysis patients," Kidney International, vol. 65, no. 5, pp. 1947-1955, 2004.

[9] G. C. Martelossi Cebinelli, K. Paiva Trugilo, S. Badaró Garcia, and K. Brajão de Oliveira, "TGF- $\beta 1$ functional polymorphisms: a review," European Cytokine Network, vol. 27, no. 4, pp. 81-89, 2016.

[10] T. Osadnik, J. K. Strzelczyk, R. Reguła et al., “The relationships between polymorphisms in genes encoding the growth factors TGF- $\beta 1$, PDGFB, EGF, bFGF and VEGF-A and the restenosis process in patients with stable coronary artery disease treated with bare metal stent," PloS One, vol. 11, no. 3, article e0150500, 2016.

[11] M. Yang, M. Zhu, L. Tang et al., "Polymorphisms of TGF $\beta-1$ and TGFBR2 in relation to coronary artery disease in a Chinese population," Clinical Biochemistry, vol. 49, no. 12, pp. 873-878, 2016.

[12] W. M. Howell, S. Ali, M. J. Rose-Zerilli, and S. Ye, "VEGF polymorphisms and severity of atherosclerosis," Journal of Medical Genetics, vol. 42, no. 6, pp. 485-490, 2005.

[13] D. R. Morris, J. V. Moxon, E. Biros, S. M. Krishna, and J. Golledge, "Meta-analysis of the association between transforming growth factor-beta polymorphisms and complications of coronary heart disease," PloS One, vol. 7, no. 5, article e37878, 2012.

[14] Y. Chen, P. T. Dawes, J. C. Packham, and D. L. Mattey, "Interaction between smoking and polymorphism in the promoter region of the VEGFA gene is associated with ischemic heart disease and myocardial infarction in rheumatoid arthritis," The Journal of Rheumatology, vol. 38, no. 5, pp. 802-809, 2011.

[15] T. Osadnik, J. Wasilewski, A. Lekston et al., "Comparison of modification of diet in renal disease and chronic kidney disease epidemiology collaboration formulas in predicting longterm outcomes in patients undergoing stent implantation due to stable coronary artery disease," Clinical Research in Cardiology, vol. 103, no. 7, pp. 569-576, 2014.

[16] P. Trzeciak, M. Gierlotka, M. Gąsior et al., "In-hospital and 12month outcomes after acute coronary syndrome treatment in patients aged $<40$ years of age (from the Polish Registry of Acute Coronary Syndromes)," The American Journal of Cardiology, vol. 114, no. 2, pp. 175-180, 2014.

[17] T. Osadnik, J. K. Strzelczyk, A. Lekston et al., "The association of functional polymorphisms in genes encoding growth factors for endothelial cells and smooth muscle cells with the severity of coronary artery disease," BMC Cardiovascular Disorders, vol. 16, no. 1, p. $218,2016$.
[18] D. J. Stekhoven and P. Bühlmann, "MissForest-nonparametric missing value imputation for mixed-type data," Bioinformatics, vol. 28, no. 1, pp. 112-118, 2012.

[19] J. R. González, L. Armengol, X. Solé et al., "SNPassoc: an R package to perform whole genome association studies," Bioinformatics, vol. 23, no. 5, pp. 654-655, 2007.

[20] D. J. Stekhoven, "MissForest: nonparametric missing value imputation using random forest," $R$ Package Version, vol. 1, no. $4,2013$.

[21] R Core Team, R: A Language and Environment for Statistical Computing, R Foundation for Statistical Computing, Vienna, Austria, 2013, http://www.R-project.org/.

[22] T. P. Smith, "Atherosclerosis and restenosis: an inflammatory issue," Radiology, vol. 225, no. 1, pp. 10-12, 2002.

[23] M. Kollum, S. Kaiser, R. Kinscherf, J. Metz, W. Kübler, and C. Hehrlein, "Apoptosis after stent implantation compared with balloon angioplasty in rabbits. Role of macrophages," Arteriosclerosis, Thrombosis, and Vascular Biology, vol. 17, no. 11, pp. 2383-2388, 1997.

[24] W. Liu, Y. Liu, H. Jiang et al., "Plasma levels of interleukin 18, interleukin 10, and matrix metalloproteinase-9 and -137G/C polymorphism of interleukin 18 are associated with incidence of in-stent restenosis after percutaneous coronary intervention," Inflammation, vol. 36, no. 5, pp. 1129-1135, 2013.

[25] R. Y. L. Zee, H. H. Hegener, A. Fernandez-Cruz, and K. Lindpaintner, "C-reactive protein gene polymorphisms and the incidence of post-angioplasty restenosis," Atherosclerosis, vol. 176, no. 2, pp. 393-396, 2004.

[26] T. Dzielski and P. Buszman, "Inflammation and restenosis following coronary stent implantation," Postepy Kardiologii Interwencyjnej, vol. 1, no. 1, pp. 67-70, 2005.

[27] A. Tedgui and Z. Mallat, "Anti-inflammatory mechanisms in the vascular wall," Circulation Research, vol. 88, no. 9, pp. 877-887, 2001.

[28] S. Redondo, E. Ruiz, C. G. Santos-Gallego, E. Padilla, and T. Tejerina, "Pioglitazone induces vascular smooth muscle cell apoptosis through a peroxisome proliferator-activated receptor-gamma, transforming growth factor-betal, and a Smad2-dependent mechanism," Diabetes, vol. 54, no. 3, pp. 811-817, 2005.

[29] S. Redondo, J. Navarro-Dorado, M. Ramajo, Ú. Medina, and T. Tejerina, "The complex regulation of TGF- $\beta$ in cardiovascular disease," Vascular Health and Risk Management, vol. 8, pp. 533-539, 2012.

[30] Y. Tang, X. Yang, R. E. Friesel, C. P. H. Vary, and L. Liaw, "Mechanisms of TGF- $\beta$-induced differentiation in human vascular smooth muscle cells," Journal of Vascular Research, vol. 48, no. 6, pp. 485-494, 2011.

[31] D. J. Grainger, L. Wakefield, H. W. Bethell, R. W. Farndale, and J. C. Metcalfe, "Release and activation of platelet latent TGF-beta in blood clots during dissolution with plasmin," Nature Medicine, vol. 1, no. 9, pp. 932-937, 1995.

[32] M. Suthanthiran, B. Li, J. O. Song et al., "Transforming growth factor-beta 1 hyperexpression in African-American hypertensives: a novel mediator of hypertension and/or target organ damage," Proceedings of the National Academy of Sciences of the United States of America, vol. 97, no. 7, pp. 3479-3484, 2000.

[33] M. Yokota, S. Ichihara, T. L. Lin, N. Nakashima, and Y. Yamada, "Association of a T29 $\rightarrow$ C polymorphism of the transforming growth factor-betal gene with genetic 
susceptibility to myocardial infarction in Japanese," Circulation, vol. 101, no. 24, pp. 2783-2787, 2000.

[34] S. Pooja, A. Francis, S. Rajender et al., "Strong impact of TGF- $\beta 1$ gene polymorphisms on breast cancer risk in Indian women: a case-control and population-based study," PloS One, vol. 8, no. 10, article e75979, 2013.

[35] S. Xu, S. Yang, G. Sun, W. Huang, and Y. Zhang, "Transforming growth factor-beta polymorphisms and serum level in the development of osteosarcoma," DNA and Cell Biology, vol. 33, no. 11, pp. 802-806, 2014.

[36] D. Kajdaniuk, B. Marek, H. Borgiel-Marek, and B. Kos-Kudła, "Transforming growth factor $\beta 1$ (TGF $\beta 1$ ) in physiology and pathology," Endokrynologia Polska, vol. 64, no. 5, pp. 384396, 2013.

[37] B. Hudzik, J. Szkodzinski, W. Romanowski et al., "No predictive value of serum interleukin- 6 and transforming growth factor-beta1 in identifying patients with a first restenosis, recurrent restenosis or a history of restenosis," European Cytokine Network, vol. 20, no. 3, pp. 135-139, 2009.

[38] J. Szkodzinski, A. Blazelonis, K. Wilczek et al., "The role of interleukin-6 and transforming growth factor-betal in predicting restenosis within stented infarct-related artery," International Journal of Immunopathology and Pharmacology, vol. 22, no. 2, pp. 493-500, 2009.

[39] I.-M. Chung, J. Kim, Y. K. Pak et al., "Blockade of TGF- $\beta$ by catheter-based local intravascular gene delivery does not alter the in-stent neointimal response, but enhances inflammation in pig coronary arteries," International Journal of Cardiology, vol. 145, no. 3, pp. 468-475, 2010.

[40] H. Tao, G. Chen, G. Cheng, and X. Shan, "The haplotype of the TGF $\beta 1$ gene associated with cerebral infarction in Chinese," The Canadian Journal of Neurological Sciences, vol. 39, no. 5, pp. 626-631, 2012.

[41] M. Cruz, J. M. Fragoso, E. Alvarez-León et al., “The TGF-B1 and IL-10 gene polymorphisms are associated with risk of developing silent myocardial ischemia in the diabetic patients," Immunology Letters, vol. 156, no. 1-2, pp. 18-22, 2013.

[42] J. M. Fragoso, J. Zuñiga-Ramos, M. Arellano-González et al., "The T29C (rs1800470) polymorphism of the transforming growth factor- $\beta 1$ (TGF- $\beta 1$ ) gene is associated with restenosis after coronary stenting in Mexican patients," Experimental and Molecular Pathology, vol. 98, no. 1, pp. 13-17, 2015.

[43] S. M. Weis and D. A. Cheresh, "Pathophysiological consequences of VEGF-induced vascular permeability," Nature, vol. 437, no. 7058, pp. 497-504, 2005.

[44] A. Hoeben, B. Landuyt, M. S. Highley, H. Wildiers, A. T. Van Oosterom, and E. A. De Bruijn, "Vascular endothelial growth factor and angiogenesis," Pharmacological Reviews, vol. 56, no. 4, pp. 549-580, 2004.

[45] A. M. Duffy, D. J. Bouchier-hayes, and J. H. Harmey, "Vascular endothelial growth factor (VEGF) and its role in nonendothelial cells: autocrine signalling by VEGF," in VEGF and Cancer, Landes Bioscience, J. H. Harmey, Ed., pp. 133144, Landes Bioscience, Georgetown, Texas, USA, 2004.

[46] M. Shahbazi, A. A. Fryer, V. Pravica et al., "Vascular endothelial growth factor gene polymorphisms are associated with acute renal allograft rejection," Journal of the American Society of Nephrology, vol. 13, no. 1, pp. 260-264, 2002.

[47] Y. Wang, Q. Huang, J. Liu et al., "Vascular endothelial growth factor A polymorphisms are associated with increased risk of coronary heart disease: a meta-analysis," Oncotarget, vol. 8, no. 18, pp. 30539-30551, 2017.

[48] T.-H. Lin, C.-L. Wang, H.-M. Su et al., "Functional vascular endothelial growth factor gene polymorphisms and diabetes: effect on coronary collaterals in patients with significant coronary artery disease," Clinica Chimica Acta, vol. 411, no. 21-22, pp. 1688-1693, 2010.

[49] G. H. Oliveira-Paula, R. Lacchini, V. Fontana, P. S. Silva, C. Biagi, and J. E. Tanus-Santos, "Polymorphisms in VEGFA gene affect the antihypertensive responses to enalapril," European Journal of Clinical Pharmacology, vol. 71, no. 8, pp. 949-957, 2015.

[50] Z. Bagyura, L. Kiss, K. Hirschberg et al., “Association between VEGF gene polymorphisms and in-stent restenosis after coronary intervention treated with bare metal stent," Disease Markers, vol. 2017, Article ID 9548612, 7 pages, 2017.

[51] Ensembl," December 2016, http://www.ensembl.org/index. html. 


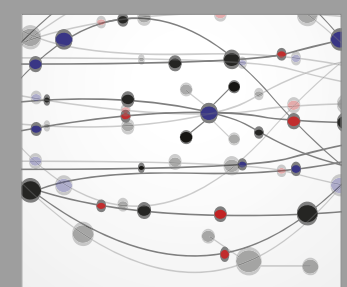

The Scientific World Journal
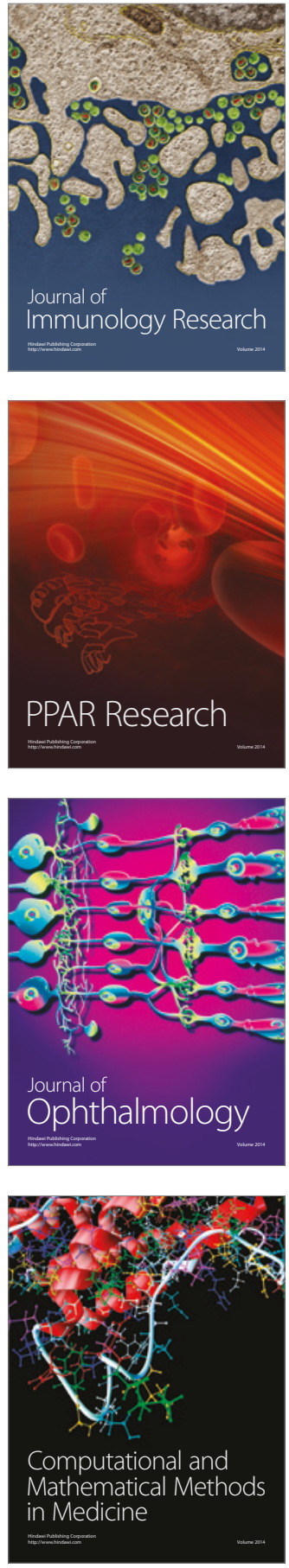

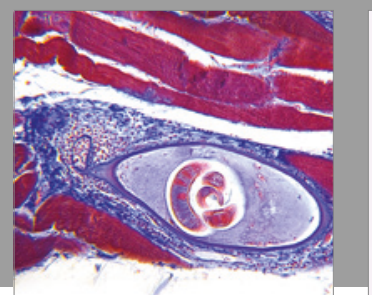

Gastroenterology Research and Practice
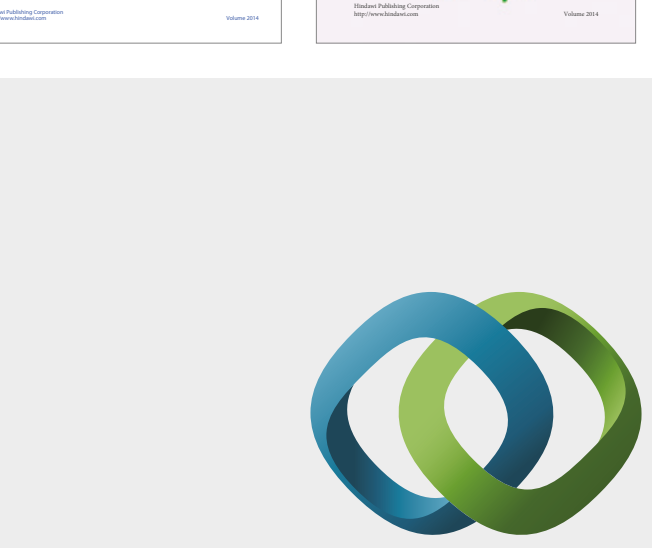

\section{Hindawi}

Submit your manuscripts at

https://www.hindawi.com
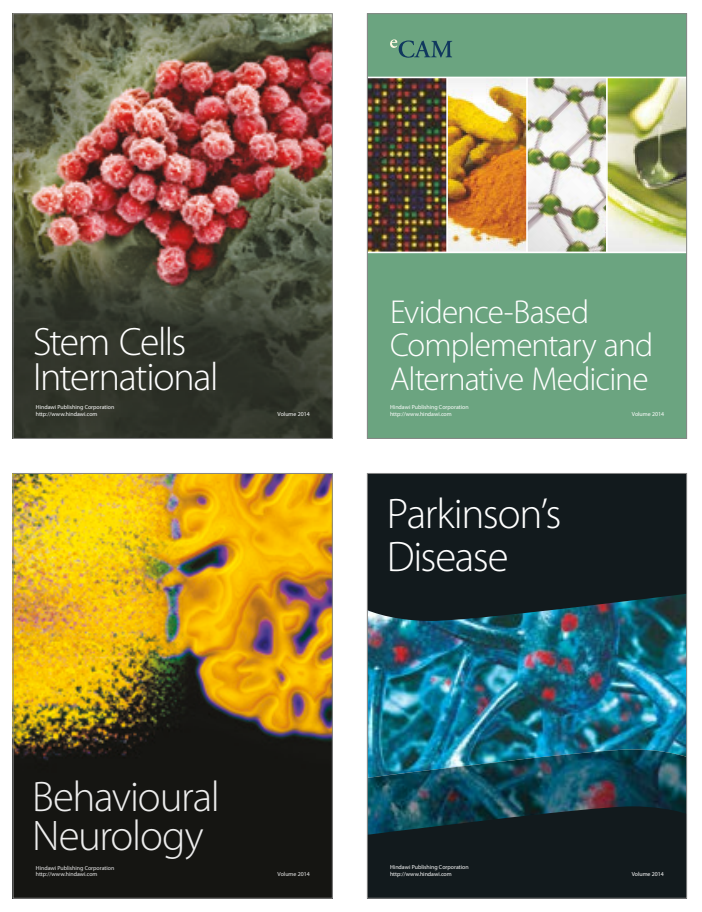
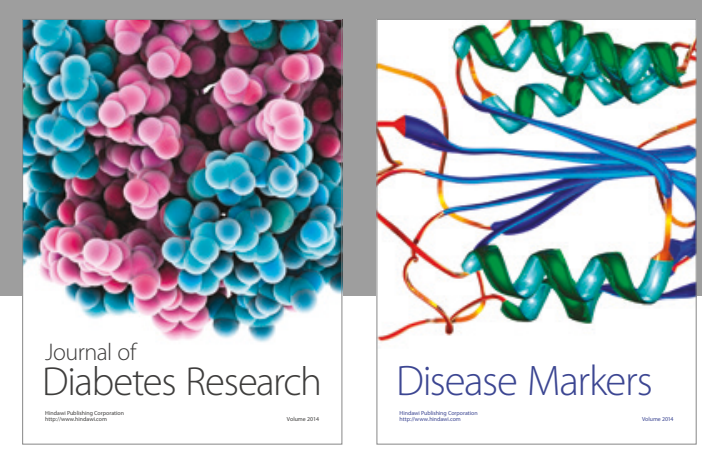

Disease Markers
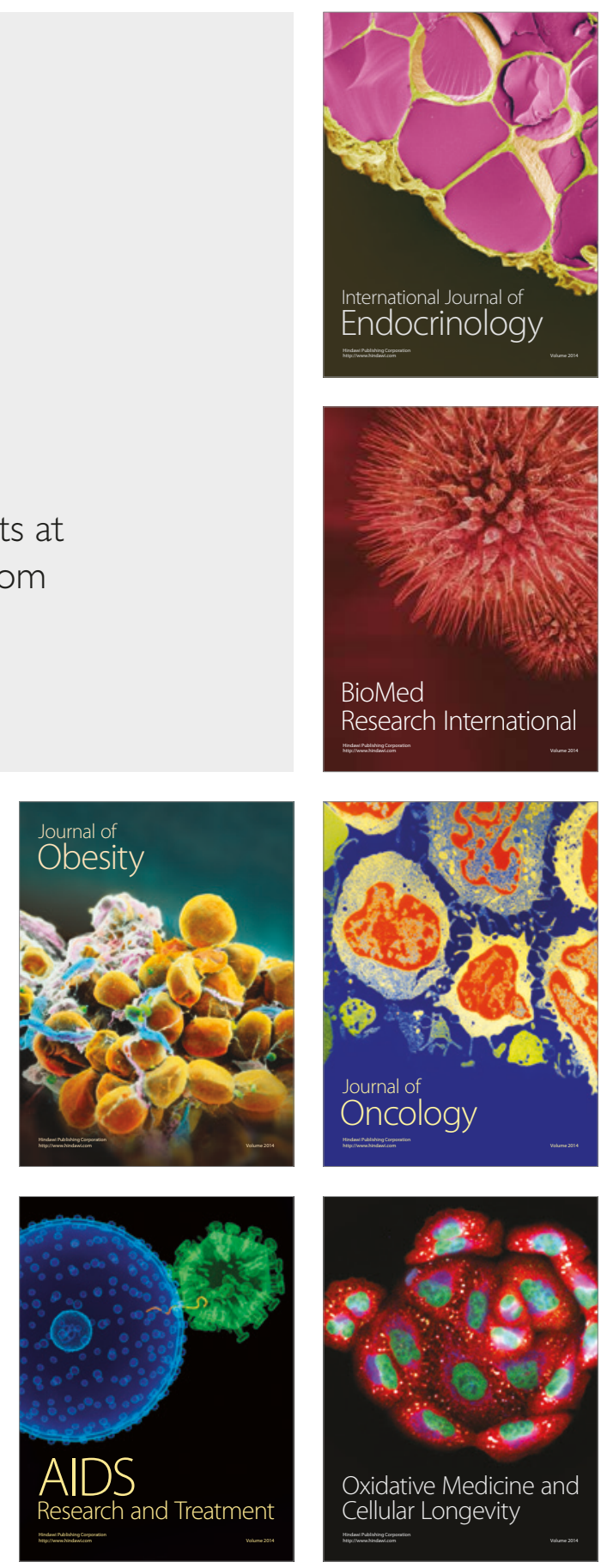